Global Journal of Pure and Applied Mathematics.

ISSN 0973-1768 Volume 17, Number 2 (2021), pp. 303-317

(C) Research India Publications

https://dx.doi.org/10.37622/GJPAM/17.2.2021.303-317

\title{
Introduction on Revised Fuzzy Modular Spaces
}

\author{
Dr. A. Muraliraj ${ }^{1}$ and R.Thangathamizh ${ }^{2 *}$ \\ ${ }^{1}$ Assistant Professor, PG \& Research Department of Mathematics, Urumu \\ Dhanalakshmi College, Bharathidasan University, Trichy, India. \\ ${ }^{2}$ Research Scholar,PG \& Research Department of Mathematics, Urumu \\ Dhanalakshmi College, Bharathidasan University, Trichy, India.
}

\begin{abstract}
The concept of Revised fuzzy modular metric space is firstly introduced in this paper. Afterwards, a Hausdorff topology induced by a $\beta$-homogeneous Revised fuzzy modular metric is defined and some related topological properties are also examined. And then, several theorems on $\mu$-completeness of the Revised fuzzy modular metric space are given. Finally, the well-known Baire's theorem and uniform limit theorem are extended to Revised fuzzy modular metric spaces.
\end{abstract}

Keywords: t-conorm, Revised fuzzy metric, Revised fuzzy modular, Uniform limit theorem.

\section{INTRODUCTION}

The notion of the fuzzy logic is introduced by Zadeh [16]. After Zadeh's result, Kramosil and Michalek [9] the notion of a fuzzy metric space was introduced and further George and Veeramani [4] \& [5] published some important results on fuzzy metric spaces. As of late In the 1960s, the concept of modular space was introduced by J. Musielak and W. Orlicz, [13]. After that Yonghong shen [15] et.al introduced the concept of fuzzy modular space in 2013. In 2018, Alexander sostak [2] defined revised fuzzy metric spaces and further in 2020 established metric spaces based on extended t-conorms [3]. Olga Grigorenka [13] et al introduced the t-conorm based

*Corresponding author (R.Thangathamizh). 
fuzzy pseudo metrics space in 2020. After that Muraliraj and ThangaThamizh [11] proved the existence of fixed points in Revised fuzzy metric spaces.

In this paper we propose the concept of Revised fuzzy modular space and examine the topological and algebraic properties.

\section{PRILIMINARY}

\section{Definition 2.1: [13]}

A real function $\rho$ on an arbitrary vector space $X$ is said to be a modular if it satisfies the following conditions:

$(\mathrm{M}-1) \rho(x)=0$ if and only if $x=\theta$ (i.e., $x$ is the null vector $\theta$ ),

$(\mathrm{M}-2) \rho(x)=\rho(-x)$,

(M-3) $\rho(\alpha x+\beta y) \leq \rho(x)+\rho(y)$ for all $x, y \in X$ and $\alpha, \beta \geq 0$ with $\alpha+\beta=$ 1.

A modular space $X_{\rho}$ is defined by a corresponding modular $\rho$, that is, $X_{\rho}=\{x \in$ $X: \rho(\lambda x) \rightarrow 0$ as $\lambda \rightarrow 0\}$.

\section{Definition 2.2: [8]}

A pair $(X, \rho)$ is called a probabilistic modular space if $X$ is a real vector space, $\rho$ is a mapping from $X$ into the set of all distribution functions (for $x \in X$, the distribution function $\rho(x)$ is denoted by $\rho_{x}$, and $\rho_{x}(t)$ is the value $\rho_{x}$ at $t \in R$ ) satisfying the following conditions:

$(\mathrm{PM}-1) \rho_{x}(0)=0$,

(PM-2) $\rho_{x}(t)=1$ for all $t>0$ if and only if $x=\theta$,

$(\mathrm{PM}-3) \rho_{-x}(t)=\rho_{x}(t)$,

$(\mathrm{PM}-4) \rho_{\alpha x+\beta y}(s+t) \geq \rho_{x}(s) \wedge \rho_{y}(t)$ for all $x, y \in X$ and $, \beta, s, t \in R_{0}^{+}, \alpha+\beta=1$.

Especially, for every $x \in X, t>0$ and $\alpha \in R \backslash\{0\}$, if

$$
\rho_{a x}(t)=\rho_{x}\left(\frac{t}{|\alpha|^{\beta}}\right), \text { where } \in(0,1],
$$

then we say that $(X, \rho)$ is $\beta$-homogeneous.

\section{We introduce}

\section{Definition 2.3: [9]}

The triple $(X, \mu, \oplus)$ is said to be a Revised fuzzy modular space (shortly, RF-modular 
space) if $X$ is a real or complex vector space, $\oplus$ is a continuous $t$-conorm, and $\mu$ is a fuzzy set on $X \times(0, \infty)$ satisfying the following conditions, for all $x, y \in X$, $s, t>0$ and $\alpha, \beta \geq 0$ with $\alpha+\beta=1$ :

$(\mathrm{RGV} 1) \mu(x, t)<1, \forall t>0$

(RGV2) $\mu(x, t)=0$ if and only if $x=y, t>0$

$(\mathrm{RGV} 3) \mu(x, t)=\mu(-x, t)$

(RGV4) $\mu(\alpha x, \beta y, s+t) \leq \mu(x, s) \oplus \mu(y, t)$

$(\mathrm{RGV} 5) \mu(x,-):(0, \infty) \rightarrow[0,1)$ is continuous.

Then $\mu$ is called a Revised fuzzy metric on $X$.

Generally, if $(X, \mu, \oplus)$ is a Revised fuzzy modular space,we say that $(\mu, \oplus)$ is a Revised fuzzy modular on $X$. Moreover, the triple $(X, \mu, \oplus)$ is called $\beta$-homogeneous if for every $x \in X, t>0$ and $\lambda \in R \backslash\{0\}$,

$$
\mu(\lambda x, t)=\mu\left(x, \frac{t}{|\lambda|^{\beta}}\right), \text { where } \beta \in(0,1] .
$$

\section{Example 2. 4.}

Let $X$ be a real or complex vector space and let $\rho$ be a modular on $X$. Take $t$ conorm $a \oplus b=a \oplus_{M} b$. For every $t \in(0, \infty)$, define $\mu(x, t)=\frac{\rho(x)}{(t+\rho(x))}$ for all $x \in X$. Then $(X, \mu, \oplus)$ is a Revised F-modular space.

\section{Remark 2.5.}

Note that the above conclusion still holds even if the $t$-conorm is replaced by $a \oplus b=a \oplus_{\rho} b$ and $a \oplus b=a \oplus_{M} b$, respectively.

\section{Example 2.6.}

Let $X=R . \rho$ is a modular on $X$, which is defined by $\rho(x)=|x|^{\beta}$, where $\beta \in$ $(0,1]$. Take $t$-conorm $a \oplus b=a \oplus_{P} b$. For every $t \in(0, \infty)$, we define

$$
\mu(x, t)=e^{-\rho(x) / t}
$$

for all $x \in X$. Then $(X, \mu, \oplus)$ is a $\beta$-homogeneous F-modular space.

\section{Proof.}

We just need to prove the condition (RGV-4) of Definition 2.3 and formula (2), because other conditions hold obviously. In the following, we first verify

$$
\mu(\alpha x+\beta y, s+t) \leq \mu(x, s) \oplus \mu(y, t),
$$

as $\alpha, \beta \geq 0$ with $\alpha+\beta=1$. 
Since $\rho$ is a modular on $X$, for all $x, y \in X$, we have

$$
\rho(\alpha x+\beta y) \leq \rho(x)+\rho(y) .
$$

Then, we can obtain

$$
\rho(\alpha x+\beta y) \leq \frac{t+s}{t} \rho(x)+\frac{t+s}{s} \rho(y),
$$

that is,

$$
\frac{1}{t+s} \rho(\alpha x+\beta y) \leq \frac{1}{t} \rho(x) \frac{1}{s}+\rho(y) .
$$

Therefore

$$
e^{-(\alpha x+\beta y) / t} \geq e^{-\rho(x) / t} \cdot e^{-\rho(y) / t}=e^{-\rho(x) / t} \oplus e^{-\rho(y) / t} .
$$

Thus, we have $\mu(\alpha x+\beta y, s+t) \leq \mu(x, s) \oplus \mu(y, t)$.

On the other hand, for all $\lambda \in R \backslash\{0\}$, since $\rho(\lambda x)=|\lambda x|^{\beta}=|\lambda|^{\beta} \cdot|x|^{\beta}=|\lambda|^{\beta} \rho(x)$, it follows that

$$
\mu(\lambda x, t)=\mu\left(x, \frac{t}{|\lambda|^{\beta}}\right) .
$$

Hence, we know that $(X, \mu, \oplus)$ is a $\beta$-homogeneous Revised F-modular space.

\section{Theorem 2.7.}

If $(X, \mu, \oplus)$ is a Revised F-modular space, then $\mu(x,-)$ is non-increasing for all $x \in$ $X$.

\section{Proof.}

Suppose that $\mu(x, t)<\mu(x, s)$ for some $t>s>0$. Without loss of generality, we can take $\alpha=1, \beta=0$, and $y=\theta$ is the null vector in $X$.

By Definition 2.3, we can obtain

$$
\begin{gathered}
\mu(x, s) \oplus \mu(\theta, t-s)=\mu(x, s) \oplus \mu(y, t-s) \geq \mu(\alpha x+\beta y, t) \\
=\mu(x, t)<\mu(x, s) .
\end{gathered}
$$

Since $\mu(\theta, t-s)=0$, we have $\mu(x, s)<\mu(x, s)$.

Obviously, this leads to a contradiction.

\section{Example 2.8.}

Let $X=R$. Take $t$-conorm $a \oplus b=a \oplus_{M} b$. For every $t \in(0, \infty)$, we define

$$
\mu(x, t)=\frac{|x|}{k+|x|},
$$

where $k>0$ is a constant. 
Here, we only show that $(x, t)$ satisfies the condition

(RGV-4) of Definition 2.3, since other conditions can be easily verified.

For every $x, y \in R$, and $\alpha, \beta \geq 0$ with $\alpha+\beta=1$. Without loss of generality, we assume that $|x| \leq|y|$. Since $|\alpha x+\beta y| \leq|y|$, we then obtain

$$
\begin{aligned}
\mu(\alpha x+\beta y, t+s) & =\frac{|\alpha x+\beta y|}{k+|\alpha x+\beta y|} \geq \frac{|y|}{k+|y|} \\
& =\max \left\{\frac{|x|}{k+|x|}, \frac{|y|}{k+|y|}\right\} \\
& =\mu(x, t) \oplus_{M} \mu(y, s)
\end{aligned}
$$

Hence $\left(\mu, \oplus_{M}\right)$ is a Revised fuzzy modular on $X$. However, if we set

$$
\mu(x, y, t)=\mu(x-y, t)=\frac{|x-y|}{k+|x-y|},
$$

it is easy to verify that $\left(\mu, \oplus_{M}\right)$ is not a Revised fuzzy metric on $X$.

\section{Topology Induced by a $\beta$-Homogeneous Revised Fuzzy Modular}

In this section, we will define a topology induced by a $\beta$-homogeneous Revised fuzzy modular and examine some topological properties. Let $\mathrm{N}$ denote the set of all positive integers.

\section{Definition 3.1.}

Let $(X, \mu, \oplus)$ be a Revised F-modular space. The $\mu$-ball $B(x, r, t)$ with center $x \in X$ and radius $r, 0<r<1, t>0$ is defined as

$$
B(x, r, t)=\{y \in X: \mu(x-y, t) \leq r\} .
$$

An element $x \in E$ is called a $\mu$-interior point of $E$ if there exist $r \in(0,1)$ and $t>$ 0 such that $B(x, r, t) \subseteq E$. Meantime, we say that $E$ is a $\mu$-open set in $X$ if and only if every element of $E$ is a $\mu$-interior point.

\section{Lemma 3.1}

If the $t$-conorm $\oplus$ is continuous, then

(L1) for every $r_{1}, r_{2} \in(0,1)$ with $r_{1}>r_{2}$, there exists $r_{3} \in(0,1)$ such that $r_{1} \oplus r_{3} \leq$ $r_{2}$,

(L2) for every $r_{4} \in(0,1)$, there exists $r_{5} \in(0,1)$ such that $r_{5} \oplus r_{5} \leq r_{4}$.

\section{Theorem 3.2.}

If $(X, \mu, \oplus)$ is a $\beta$-homogeneous Revised F-modular space, then

$$
B\left(x, r, \frac{t}{2}\right) \subset B\left(x, r, \frac{t}{2^{\beta+1}}\right) .
$$




\section{Proof.}

By Theorem 2.7, for every $r \in(0,1)$ and $t>0$, since $\mu\left(x-y, \frac{t}{2}\right) \leq \mu\left(x-y, \frac{t}{2^{\beta+1}}\right)$, it is obvious that $\left.\left\{y \in X: \mu\left(x-y, \frac{t}{2^{\beta+1}}\right)<r\right)\right\} \subset\left\{y \in X: \mu\left(x-y, \frac{t}{2}\right)<r\right\}$.

\section{Theorem 3.3.}

Let $(\mathrm{X}, \mu, \oplus)$ be a $\beta$-homogeneous Revised Fuzzy modular space. Every $\mu$-ball $B(x, r, t)$ in $(X, \mu, \oplus)$ is a $\mu$-open set.

\section{Proof.}

By Definition 10, for every $y \in B(x, r, t)$, wehave $\mu(x-y, t)<r$.

Without loss of generality, we may assume that $t=2 t_{1}$.

Since $\mu(x-y,-)$ is continuous, there exists an $\epsilon_{y}>0$ such that $\mu\left(x-y, \frac{\left(t_{1}-\epsilon\right)}{2^{\beta-1}}\right)<r$ for some $\epsilon>0$ with $\frac{\left(t_{1}-\epsilon\right)}{2^{\beta-1}}>0$ and $\frac{\epsilon}{2^{\beta-1}} \in\left(0, \epsilon_{y}\right)$. Set $r_{0}=\mu\left(x-y, \frac{\left(t_{1}-\epsilon\right)}{2^{\beta-1}}\right)$.

Since $r_{0}<r$, there exists an $s \in(0,1)$ such that $r_{0}<s<r$. According to Lemma 11 , we can find an $r_{1} \in(0,1)$ such that $r_{0} \oplus r_{1}<s$.

Next, we show that $B(x, r, 2 t 1) \subset B\left(y, r_{1}, \frac{\epsilon}{2^{\beta-1}}\right)$. For every $z \in B\left(y, r_{1}, \frac{\epsilon}{2^{\beta-1}}\right)$, we have $\mu B\left(y-z, \frac{\epsilon}{2^{\beta-1}}\right)<r_{1}$

Therefore,

$$
\begin{aligned}
\mu(x-z, t)= & \mu\left(x-z, 2 t_{1}\right) \leq \mu\left(2(x-y), 2\left(t_{1}-\epsilon\right)\right) \oplus \mu(2(y-z), 2 \epsilon) \\
& =\mu\left(x-y, \frac{\left(t_{1}-\epsilon\right)}{2^{\beta-1}}\right) \oplus \mu\left(\frac{\epsilon}{2^{\beta-1}}\right) \\
& \leq r_{0} \oplus r_{1} \leq s<r .
\end{aligned}
$$

Thus $z \in B(x, r, t)$ and hence $B(x, r, t) \subset B\left(y, r_{1}, \frac{\epsilon}{2^{\beta-1}}\right)$.

\section{Theorem 3.4.}

Let $(X, \mu, \oplus)$ be a $\beta$-homogeneous Revised Fuzzy modular space. Define

$T_{\mu}=\{A \subset X: x \in A$ if and only if there exist $t>0$ and $r \in(0,1)$

such that

$$
B(x, r, t) \subset A\} .
$$

Then $T_{\mu}$ is a topology on $X$. 


\section{Proof.}

The proof will be divided into three parts.

(i) Obviously, $0, X \in T_{\mu}$

(ii) Suppose that $A, B \in T_{\mu}$. If $x \in A \cap B$, then $x \in A$ and $x \in B$.

Therefore, there exist $0<r_{1}, r_{2}<1$ and $t_{1}, t_{2}>0$ such that $B\left(x, r_{1}, t_{1}\right) \subset A$ and $B\left(x, r_{2}, t_{2}\right) \subset B$. Set $r=\max \left\{r_{1}, r_{2}\right\}, t=\max \left\{t_{1}, t_{2}\right\}$. Now, we claim that $B(x, r, t) \subset B\left(x, r_{1}, t_{1}\right)$.

If $y \in B(x, r, t)$, then we know that $\mu(x-y, t)<r$.

According to Theorem 2.7, we can obtain

$$
\mu\left(x-y, t_{1}\right) \geq \mu(x-y, t)<r \leq r_{1} .
$$

Thus, $y \in B\left(x, r_{1}, t_{1}\right)$, that is, $B(x, r, t) \subset B\left(x, r_{1}, t_{1}\right)$.

Similarly, $B(x, r, t) \subset B\left(x, r_{2}, t_{2}\right)$.

Hence, $B(x, r, t) \subset B\left(x, r_{1}, t_{1}\right) \cap B\left(x, r_{2}, t_{2}\right) \subset A \cap B$. That is to say, $A \cap B \in T_{\mu}$.

(iii) Suppose that $T_{\mu}{ }^{\prime} \subset T_{\mu}$. If $x \in \cup_{A \in T_{\mu}{ }^{\prime}} A$, then there exists $U \in T_{\mu}{ }^{\prime}$ such that $x \in U$. Since $U \in T \mu$, there exist $0<r<1$ and $t>0$ such that $B(x, r, t) \subset U \subset \mathrm{U}_{A \in T_{\mu}}{ }^{A}$. Hence, $\mathrm{U}_{A \in T_{\mu}}{ }^{\prime} A \in T_{\mu}$.

Obviously, if we take $=t=(1 / n)(n=1,2,3, . .$.$) , then the family of \mu$-ball $B(x, 1 / n, 1 / n),(n=1,2,3, \ldots)$ constitutes a countable local base at $x$. Therefore, we can obtain Theorem 3.5.

\section{Theorem 3.5.}

The topology $T_{\mu}$ induced by a $\beta$-homogeneous RF-modular space is first countable.

\section{Theorem 3.6.}

Every $\beta$-homogeneous RF-modular space is Hausdorff.

\section{Proof.}

For the $\beta$-homogeneous RF-modular space $(X, \mu, \oplus)$, let $x$ and $y$ be two distinct points in $X$. By Definition 3, we can easily obtain $0<\mu(x-y, t)<1$ for all $t>$ 0 .

Set $r=\mu(x-y, t)$. According to Lemma 11, for every $r_{0} \in(r, 1)$, there exists $r_{1} \in(0,1)$ such that $r_{1} \oplus r_{1} \leq r_{0}$.

Next, we consider the $\mu$-balls $B\left(x, r_{1}, \frac{t}{2^{\beta+1}}\right)$ and $B\left(y, r_{1}, \frac{t}{2^{\beta+1}}\right)$ and then show that $B\left(x, r_{1}, \frac{t}{2^{\beta+1}}\right) \cap B\left(y, r_{1}, \frac{t}{2^{\beta+1}}\right)=0$ using reduction to absurdity. If there 
exists $z \in B\left(x, r_{1}, \frac{t}{2^{\beta+1}}\right) \cap B\left(y, r_{1}, \frac{t}{2^{\beta+1}}\right)$, then

$$
\begin{aligned}
r & =\mu(x-y, t) \leq \mu\left(2(x-z), \frac{t}{2}\right) \oplus \mu\left(2(z-y), \frac{t}{2}\right) \\
& =\mu\left(x-z, \frac{t}{2^{\beta+1}}\right) \oplus \mu\left(z-y, \frac{t}{2^{\beta+1}}\right) \leq r_{1} \oplus r_{1} \leq r_{0},
\end{aligned}
$$

which is a contradiction. Hence $(X, \mu, \oplus)$ is Hausdorff.

In order to obtain some further properties, several basic notions derived from general topology are introduced in the RF-modular space.

\section{Definition 3.7.}

Let $(X, \mu, \oplus)$ be a RF-modular space.

(i) A sequence $\left\{x_{n}\right\}$ in $X$ is said to be $\mu$-convergent to a point $x \in X$, denoted by $\quad x_{n} \stackrel{\mu}{\rightarrow} x$, if for every $r \in(0,1)$ and $t>0$, there exists $n_{0} \in N$ such that $x_{n} \in B(x, r, t)$ for all $n \geq n_{0}$.

(ii) A subset $A \subset X$ is called $\mu$-bounded if and only if there exist $t>0$ and $r \in(0,1)$ such that $\mu(x, t)<r$ for all $x \in A$.

(iii) A subset $B \subset X$ is called $\mu$-compact if and only if every $\mu$-open cover of $B$ has a finite subcover (or equivalently, every sequence in $B$ has a $\mu$-convergent subsequence in $B)$.

(iv) A subset $C \subset X$ is called a $\mu$-closed if and only if for every sequence $\left\{x_{n}\right\} \subset C$, $x_{n} \stackrel{\mu}{\rightarrow} x$ implies $x \in C$.

\section{Theorem 3.8.}

Every $\mu$-compact subset $A$ of a $\beta$-homogeneous F-modular space $(X, \mu, \oplus)$ is $\mu$-bounded.

\section{Proof.}

Suppose that $A$ is a $\mu$-compact subset of the given $\beta$-homogeneous RF-modular space $(X, \mu, \oplus)$. Fix $t>0$ and $r \in(0,1)$, it is easy to see that the family of $\mu$-ball $\left\{B\left(x, r_{1}, \frac{t}{2^{\beta+1}}\right): x \in A\right\}$ is a $\mu$-open cover of $A$. Since $A$ is $\mu$-compact, there exist $x_{1}, x_{2}, \ldots, x_{n} \in A$ such that $A \subseteq \bigcup_{i=1}^{n} B\left(x_{i}, r, \frac{t}{2^{\beta+1}}\right)$. For every $x \in A$, there exists $i$ such that $x \in B\left(x_{i}, r, \frac{t}{2^{\beta+1}}\right)$. Therefore, we have $\mu\left(x-x_{i}, \frac{t}{2^{\beta+1}}\right)<r$.

Set $\alpha=\max \left\{\mu\left(x_{i}, \frac{t}{2^{\beta+1}}\right): 1 \leq i \leq n\right\}$. Clearly, we know that $\alpha>0$. 
Thus, we have

$$
\begin{aligned}
\mu(x, t) & =\mu\left(\left(x-x_{i}\right)+x_{i}, t\right) \leq \mu\left(2\left(x-x_{i}\right), \frac{t}{2}\right) \oplus \mu\left(2 x_{i}, \frac{t}{2}\right) \\
& =\mu\left(x-x_{i}, \frac{t}{2^{\beta+1}}\right) \oplus \mu\left(x_{i}, \frac{t}{2^{\beta+1}}\right) \leq r \oplus \alpha<s
\end{aligned}
$$

for some $s \in(0,1)$. This shows that $A$ is $\mu$-bounded.

\section{Theorem 3.9.}

Let $(X, \mu, \oplus)$ be a $\beta$-homogeneous RF-modular space, and let $T_{\mu}$ be the topology induced by the $\beta$-homogeneous modular. Then for a sequence $\left\{x_{n}\right\}$ in $X, x_{n} \stackrel{\mu}{\rightarrow} x$ if and only if $\mu\left(x-x_{n}, t\right) \rightarrow 0$ as $n \rightarrow \infty$.

\section{Proof.}

Fix $t>0$. Suppose that $x_{n} \stackrel{\mu}{\rightarrow} x$. Then for every $r \in(0,1)$, there exists $n_{0} \in N$ such that $x_{n} \in B(x, r, t)$ for all $n \geq n_{0}$. Namely, $\mu\left(x_{n}-x, t\right)<r$ for all $n \geq n_{0}$. Thus, we have $\mu\left(x_{n}-x, t\right)<r$ for all $n \geq n_{0}$. Because $r$ is arbitrary, we can verify that $\mu\left(x_{n}-x, t\right) \rightarrow 0$ as $n \rightarrow \infty$.

On the other hand, if for every $t>0, \mu\left(x-x_{n}, t\right) \rightarrow 0$ as $n \rightarrow \infty$, then for every $r \in(0,1)$, there exists $n_{0} \in N$ such that $\mu\left(x_{n}-x, t\right)<r$ for all $n \geq n_{0}$. Therefore, we know that $\mu\left(x_{n}-x, t\right)<r$ for all $n \geq n_{0}$.. Thus $x_{n} \in B(x, r, t)$ for all $n \geq n_{0}$, and hence $x_{n} \stackrel{\mu}{\rightarrow} x$ as $n \rightarrow \infty$.

\section{4. $\mu$-COMPLETENESS OF A FUZZY MODULAR SPACE}

In this section, we will establish some related theorems of $\mu$-completeness of a Revised fuzzy modular space.

\section{Definition 4.1.}

Let $(X, \mu, \oplus)$ be a RF-modular space.

(i) A sequence $\left\{x_{n}\right\}$ in $X$ is a $\mu$-Cauchy sequence if and only if for every $\epsilon \in(0,1)$ and $t>0$, there exists $n_{0} \in N$ such that $\mu\left(x_{m}-x_{n}, t\right)<\epsilon$ for all $m, n \geq n_{0}$.

(ii) The F-modular space $(X, \mu, \oplus)$ is called $\mu$-complete if every $\mu$-Cauchy sequence is $\mu$-convergent.

\section{Theorem 4.2.}

Let $\left(X, \mu, \oplus_{\mu}\right)$ be a $\beta$-homogeneous F-modular space. Then every $\mu$-convergent sequence $\left\{x_{n}\right\}$ in $X$ is a $\mu$-Cauchy sequence. 
Proof.

Suppose that the sequence $\left\{x_{n}\right\} \mu$-converges to $x \in X$. Therefore, for every $\epsilon \in$ $(0,1)$ and $t>0$, there exists $n_{0} \in N$ such that $\mu\left(x_{n}-x, \frac{t}{2^{\beta^{+1}}}\right)$ for all $n \geq n_{0}$. For all $m, n \geq n_{0}$, we have

$$
\begin{aligned}
\mu\left(x_{m}-x_{n}, t\right) & \leq \mu\left(2\left(x_{m}-x\right), \frac{t}{2}\right) \oplus_{\mathrm{M}} \mu\left(2\left(x_{n}-x\right), \frac{t}{2}\right) \\
& \leq \mu\left(x_{m}-x, \frac{t}{2^{\beta+1}}\right) \oplus_{\mathrm{M}} \mu\left(x_{n}-x, \frac{t}{2^{\beta+1}}\right) \\
& \leq \epsilon \oplus_{\mathrm{M}} \epsilon=\epsilon
\end{aligned}
$$

Hence $\left\{x_{n}\right\}$ is a $\mu$-Cauchy sequence in $X$.

\section{Remark 4.3.}

The proof of Theorem 4.2 shows that, in the RF-modular space, a $\mu$-convergent sequence is not necessarily a $\mu$-Cauchy sequence. However, the $\beta$-homogeneity and the choice of triangular conorms are essential to guarantee the establishment of theorem.

\section{Theorem 4.4.}

Every $\mu$-closed subspace of $\mu$-complete RF-modular space is $\mu$-complete.

\section{Proof.}

From Definition 4.1, it is evident to see that the theorem holds.

\section{Theorem 4.5.}

Let $(X, \mu, \oplus)$ be a $\beta$-homogeneous RF-modular space, and let $Y$ be a subset of $X$. If every $\mu$-Cauchy sequence of $Y$ is $\mu$-convergent in $X$, then every $\mu$-Cauchy sequence of $\bar{Y}$ is also $\mu$-convergent in $X$, where $\bar{Y}$ denotes the $\mu$-closure of $Y$.

\section{Proof.}

Suppose that the sequence $\left\{x_{n}\right\}$ is a $\mu$-Cauchy sequence of $\bar{Y}$.Therefore, for every $n \in N$ and $t>0$, there exists $y_{n} \in Y$ such that $\mu\left(x_{n}-y_{n}, \frac{t}{4^{\beta+1}}\right)<\left(\frac{1}{(n+1)}\right)$. According to Theorem 2.7, we have $\mu\left(x_{n}-y_{n}, \frac{t}{2^{\beta+1}}\right)<\left(\frac{1}{(n+1)}\right)$. In addition, for every $r \in(0,1)$ and $t>0$, there exists an $n_{0} \in N$ such that $\mu\left(x_{n}-x_{m}, \frac{t}{4^{\beta+1}}\right)<r$ for all $m, n \geq n_{0}$. That is to say, $\mu\left(x_{n}-x_{m}, \frac{t}{4^{\beta+1}}\right) \rightarrow 0$ as $m, n \rightarrow \infty$. Next, we will show that the sequence $\left\{y_{n}\right\}$ is a $\mu$-Cauchy sequence of $Y$. For every $m, n \geq n_{0}$, 
we have

$$
\begin{aligned}
\mu\left(y_{n}-y_{m}, t\right) & \leq \mu\left(2\left(y_{n}-x_{n}\right), \frac{t}{2}\right) \oplus \mu\left(2\left(x_{n}-y_{m}\right), \frac{t}{2}\right) \\
& \leq \mu\left(2\left(y_{n}-x_{n}\right), \frac{t}{2}\right) \oplus \mu\left(4\left(x_{n}-x_{m}\right), \frac{t}{4}\right) \oplus \mu\left(4\left(x_{m}-y_{m}\right), \frac{t}{4}\right) \\
& \leq \mu\left(y_{n}-x_{n}, \frac{t}{2^{\beta+1}}\right) \oplus \mu\left(x_{n}-x_{m}, \frac{t}{4^{\beta+1}}\right) \oplus \mu\left(x_{m}-y_{m}, \frac{t}{4^{\beta+1}}\right) \\
& <\left(\frac{1}{(n+1)}\right) \oplus r \oplus\left(\frac{1}{(m+1)}\right) .
\end{aligned}
$$

Since $t$-conorm $\oplus$ is continuous, it follows that $\mu\left(y_{n}-y_{m}, t\right) \rightarrow 0$ as $m, n \rightarrow \infty$.

Now, we assume that the sequence $\left\{y_{n}\right\} \mu$-converges to $x \in X$. Thus, for every $\epsilon \in(0,1)$ and $t>0$, there exists an $n_{1} \in N$ such that $\mu\left(x-y_{n}, \frac{t}{2^{\beta+1}}\right)<\epsilon$ for all $n \geq n_{1}$. Therefore, for all $n \geq n_{1}$, we can obtain

$$
\begin{aligned}
\mu\left(x_{n}-x, t\right) & \leq \mu\left(2\left(x_{n}-y_{n}\right), \frac{t}{2}\right) \oplus \mu\left(2\left(y_{n}-x_{n}\right), \frac{t}{2}\right) \\
& \leq \mu\left(2\left(x_{n}-y_{n}\right), \frac{t}{2^{\beta+1}}\right) \oplus \mu\left(2\left(y_{n}-x_{n}\right), \frac{t}{2^{\beta+1}}\right) \\
& <\epsilon \oplus\left(\frac{1}{(n+1)}\right) .
\end{aligned}
$$

According to the arbitrary of $\epsilon$ and by letting $n \rightarrow \infty$, it follows that $\lim _{n \rightarrow \infty} \mu\left(x_{n}-x, t\right)=0$. That is, an arbitrary $\mu$-Cauchy sequence $\left\{x_{n}\right\}$ of $\bar{Y}$ $\mu$-converges to $x \in X$. The proof of the theorem is now completed.

\section{Theorem 4.6.}

Let $(X, \mu, \oplus)$ be a $\beta$-homogeneous RF-modular space, and let $Y$ be a dense subset of $X$. If every $\mu$-Cauchy sequence of $Y$ is $\mu$-convergent in $X$, then the $\beta$-homogeneous RF-modular space $(X, \mu, \oplus)$ is $\mu$-complete.

\section{Proof.}

It follows from Theorem 4.5.

\section{BAIRE'S THEOREM AND UNIFORM LIMIT THEOREM}

\section{Theorem 5.1 (Baire's theorem).}

Let $U_{n}(n=1,2, \ldots)$ be a countable number of $\mu$-dense and $\mu$-open sets in the $\mu$-complete $\beta$-homogeneous RF-modular space $\left(X, \mu, \oplus_{\mathrm{M}}\right)$. Then $\bigcap_{n=1}^{\infty} U_{n}$ is $\mu$-dense in $X$. 


\section{Proof.}

First of all, if $B(x, r, 2 t)$ is a $\mu$-ball in $X$ and $y$ is an arbitrary element of it, then we know that $\mu(x-y, 2 t)<r$. Since $\mu(x-y,-)$ is continuous, there exists an $\epsilon_{y}>0$ such that $\mu\left(x-y, \frac{(t-\epsilon)}{2^{\beta-1}}\right)<r$ for some $\epsilon>0$ with $\frac{(t-\epsilon)}{2^{\beta-1}}>0$ and $\frac{\epsilon}{2^{\beta-1}} \in\left(0, \epsilon_{y}\right)$. Choose $r^{\prime} \in(0, r), \frac{\epsilon}{2^{\beta-1}} \in\left(0, \epsilon_{y}\right)$ and $z \in \overline{B\left(y, r^{\prime}, \frac{\epsilon}{4^{\beta}}\right)}$, there exists a sequence $\left\{z_{n}\right\}$ in $\overline{B\left(y, r^{\prime}, \frac{\epsilon}{4 \beta}\right)}$ such that $z_{n} \stackrel{\mu}{\rightarrow} z$ and hence we have

$$
\begin{aligned}
\mu\left(z-y, \frac{\epsilon}{2^{\beta-1}}\right) & \leq \mu\left(2\left(z-z_{n}\right), \frac{\epsilon}{2^{\beta}}\right) \oplus_{\mathrm{M}} \mu\left(2\left(z_{n}-y\right), \frac{\epsilon}{2^{\beta}}\right) \\
& =\mu\left(z-z_{n}, \frac{\epsilon}{4^{\beta}}\right) \oplus_{\mathrm{M}} \mu\left(z_{n}-y, \frac{\epsilon}{4^{\beta}}\right) \\
& <r
\end{aligned}
$$

for some $n \in N$. Therefore, we can obtain

$$
\begin{gathered}
\mu(x-z, 2 t)=\mu(2(z-y), 2 \epsilon) \oplus_{\mathrm{M}} \mu(2(x-y), 2(t-\epsilon)) \\
=\mu\left(z-y, \frac{\epsilon}{2^{\beta-1}}\right) \oplus_{\mathrm{M}} \mu\left(x-y, \frac{t-\epsilon}{2^{\beta-1}}\right) \\
>r \oplus_{\mathrm{M}} r=r .
\end{gathered}
$$

This shows that $\overline{B\left(y, r^{\prime}, \frac{\epsilon}{4^{\beta}}\right)} \subseteq B(x, r, 2 t)$. It means that if $A$ is a nonempty $\mu$-open set of $X$, then $A \cap U_{1}$ is nonempty and $\mu$-open. Now, let $x_{1} \in A \cap U_{1}$, there exist $r_{1} \in(0,1)$ and $t_{1}>0$ such that $B\left(x_{1}, r_{1}, \frac{t_{1}}{2^{\beta-1}}\right) \subseteq A \cap U_{1}$. Choose $r_{1}^{\prime}<r_{1}$ and $t_{1}^{\prime}=\max \left\{t_{1}, 1\right\}$ such that $\left(x_{1}, r_{1}^{\prime}, \frac{t_{1}^{\prime}}{2^{\beta-1}}\right) \subseteq A \cap U_{1}$. Since $U_{2}$ is $\mu$-dense in $X$, we can obtain $B\left(x_{1}, r_{1}^{\prime}, \frac{t_{1}^{\prime}}{2^{\beta-1}}\right) \subseteq A \cap U_{2} \neq \emptyset$. Let $x_{2} \in B\left(x_{1}, r_{1}^{\prime}, \frac{t_{1}^{\prime}}{2^{\beta-1}}\right) \cap U_{2}$, there exist $r_{2} \in\left(0, \frac{1}{2}\right)$ and $t_{2}>0$ such that $B\left(x_{2}, r_{2}, \frac{t_{1}}{2^{\beta-1}}\right) \subseteq B\left(x_{1}, r_{1}^{\prime}, \frac{t_{1}^{\prime}}{2^{\beta-1}}\right) \cap U_{2}$. Choose $r_{2}^{\prime}<r_{1} \quad$ and $t_{1}^{\prime}=\max \left\{t_{1}, \frac{1}{2}\right\}$ such that $\overline{B\left(x_{2}, r_{2}^{\prime}, \frac{t_{2}^{\prime}}{2^{\beta-1}}\right)} \subseteq A \cap U_{2}$. By induction, we can obtain a sequence $\left\{x_{n}\right\}$ in $X$ and two sequence $\left\{r_{n}^{\prime}\right\},\left\{t_{n}^{\prime}\right\}$ such that $0<r_{n}^{\prime}<\frac{1}{n}, 0<t_{n}^{\prime}<\frac{1}{n}$ and $\overline{B\left(x_{n}, r_{n}^{\prime}, \frac{t_{n}^{\prime}}{2^{\beta-1}}\right)} \subseteq A \cap U_{n}$.

Next,we show that $\left\{x_{n}\right\}$ is a $\mu$-Cauchy sequence. For given $t>0$ and $r \in(0,1)$, we can choose $k \in N$ such that $2 t_{k}^{\prime}<t$ and $r_{k}^{\prime}<r$. Then for $m, n \geq k$, since $x_{m}, x_{n} \in B\left(x_{k}, r_{k}^{\prime}, \frac{t_{k}^{\prime}}{2^{\beta-1}}\right)$, 
we have

$$
\begin{aligned}
\mu\left(x_{m}-x_{n}, 2 t\right) & \leq \mu\left(x_{m}-x_{n}, 4 t_{k}^{\prime}\right) \\
& \leq \mu\left(2\left(x_{m}-x_{k}\right), t_{k}^{\prime}\right) \oplus_{\mathrm{M}} \mu\left(2\left(x_{n}-x_{k}\right), t_{k}^{\prime}\right) \\
& =\mu\left(x_{m}-x_{k}, \frac{t_{k}^{\prime}}{2^{\beta-1}}\right) \oplus_{\mathrm{M}} \mu\left(x_{m}-x_{k}, \frac{t_{k}^{\prime}}{2^{\beta-1}}\right) \\
& \leq r_{k}<r
\end{aligned}
$$

According to the arbitrary of $t$, it follows that $\left\{x_{n}\right\}$ is a $\mu$-Cauchy sequence. Since $X$ is $\mu$-complete, there exists $x \in X$ such that $x_{n} \stackrel{\mu}{\rightarrow} x$. But $x_{n} \in B\left(x_{k}, r_{k}^{\prime}, \frac{t_{k}^{\prime}}{2^{\beta-1}}\right)$ for all $n \geq k$, and therefore $\quad x \in \overline{B\left(x_{k}, r_{k}^{\prime}, \frac{t_{k}^{\prime}}{2^{\beta-1}}\right)} \subseteq A \cap U_{k}$ for all $k$. Thus $A \cap\left(\cap_{n=1}^{\infty} U_{n}\right) \neq \emptyset$. Hence $\bigcap_{n=1}^{\infty} U_{n}$ is $\mu$-dense in $X$.

\section{Definition 5.2.}

Let $X$ be any nonempty set and let $(Y, \mu, \oplus)$ be a RF-modular space. A sequence $\left\{f_{n}\right\}$ of functions from $X$ to $Y$ is said to $\mu$-converge uniformly to a function $f$ from $X$ to $Y$ if given $t>0$ and $r \in(0,1)$; there exists $n_{0} \in N$ such that $\mu\left(f_{n}(x)-f(x), t\right)<r$ for all $n \geq n_{0}$ and for every $x \in X$.

\section{Theorem 5.3 (Uniform limit theorem).}

Let $f_{n}: X \rightarrow Y$ be a sequence of continuous functions from a topological space $X$ to a $\beta$-homogeneous RF-modular space $(Y, \mu, \oplus)$. If $\left\{f_{n}\right\} \quad \mu$-converges uniformly to $f: X \rightarrow Y$, then $f$ is continuous.

\section{Proof.}

Let $V$ be a $\mu$-open set of and $x_{0} \in f^{-1}(V)$. Since $V$ is $\mu$-open, there exist $r \in(0,1)$ and $t>0$ such that $B\left(f\left(x_{0}\right), r, t\right) \subset V$. Owing to $r \in(0,1)$, we can choose $s \in(0,1)$ such that $s \oplus s \oplus s<r$.

Since $\left\{f_{n}\right\} \mu$-converges uniformly to $f$, given $s \in(0,1)$ and $t>0$, there exists $n_{0} \in N$ such that $\mu\left(f_{n}(x)-f(x), \frac{t}{4^{\beta+1}}\right)<s$ for all $n \geq n_{0}$ and for every $x \in X$. Moreover, $f_{n}$ is continuous for every $n \in N$, there exists a neighborhood $U$ of $x_{0}$ such that $f_{n}(U) \subset B\left(f_{n}\left(x_{0}\right), s, \frac{t}{4^{\beta+1}}\right)$. 
Therefore, we know that $\mu\left(f_{n}(x)-f_{n}\left(x_{0}\right), \frac{t}{4^{\beta+1}}\right)<s$ for every $x \in U$. Thus, we have

$$
\begin{aligned}
\mu(f(x) & \left.-f\left(x_{0}\right), t\right) \leq \mu\left(2\left(f(x)-f_{n}(x)\right), \frac{t}{2}\right) \oplus \mu\left(2\left(f_{n}(x)-f\left(x_{0}\right)\right), \frac{t}{2}\right) \\
& =\mu\left(f(x)-f_{n}(x), \frac{t}{2^{\beta+1}}\right) \oplus \mu\left(2\left(f_{n}(x)-f\left(x_{0}\right)\right), \frac{t}{2^{\beta+1}}\right) \\
& \leq \mu\left(f(x)-f_{n}(x), \frac{t}{2^{\beta+1}}\right) \oplus \mu\left(2\left(f_{n}(x)-f\left(x_{0}\right)\right), \frac{t}{2^{\beta+2}}\right) \oplus \mu\left(2\left(f_{n}\left(x_{0}\right)-f\left(x_{0}\right)\right), \frac{t}{2^{\beta+2}}\right) \\
& =\mu\left(f(x)-f_{n}(x), \frac{t}{2^{\beta+1}}\right) \oplus \mu\left(f_{n}(x)-f\left(x_{0}\right), \frac{t}{4^{\beta+2}}\right) \oplus \mu\left(f_{n}\left(x_{0}\right)-f\left(x_{0}\right), \frac{t}{4^{\beta+2}}\right) \\
& \leq \oplus \mathrm{s} \oplus \mathrm{s} \oplus s<r .
\end{aligned}
$$

This shows that $f(x) \in B(f(x 0), r, t) \subset V$. Hence $(U) \subset V$; that is, $f$ is continuous.

\section{Remark 5.4.}

All the results in this paper are still valid if the condition (RFM-5) in Definition 3 is replaced by Right continuity.

\section{REFERENCES}

[1] Adam Grabowski "Basic Formal Properties of Triangular Norms and Conorms "Formalized Mathematics Vol. 25, No. 2, Pages 93-100, 2017 DOI: 10.1515/ forma-2017-0009.

[2] Alexander Sostak "George-Veeramani Fuzzy Metrics Revised" Axioms 2018,7,60; doi:10.3390/axioms7030060.

[3] Alexander Sostak and Tarkan Öner On Metric-Type Spaces Based on Extended T-Conorms Mathematics 2020, 8, 1097; doi:10.3390/math8071097.

[4] George .A and Veeramani.P, “On some results in fuzzy metric spaces”, Fuzzy Sets and Systems, 64 (1994), 395-399.

[5] George .A and Veeramani.P, "On some results of analysis for fuzzy metric spaces”, Fuzzy Sets and Systems, 90 (1997),365-399.

[6] Grabiec.M, "Fixed points in fuzzy metric spaces", Fuzzy Sets and Systems, 27 (1988), 385-389.

[7] Gregori.V, Samuel Morillas, and AlmanzorSapena, "Examples of fuzzy metrics and applications”, Fuzzy Sets and Systems, 170 (2011), 95-111. 
[8] W. M. Kozłowski, "Notes on modular function spaces"Commentationes Mathematicae, vol. 28, no. 1, pp. 87-100, 1988.

[9] Kramosil.I and Michalek.J, "Fuzzy metric and Statistical metric spaces", Kybernetica, 11(1975),326-334.

[10] Khurshid Ahmad, Andrea Mesiarová-Zemánková, "Chosing t-Norms and $t$ Conorms for Fuzzy Controllers, ", Fourth International Conference on Fuzzy Systems and Knowledge Discovery, Haikou, Hainan, China, 24-27 August 2007, edited by Jingsheng Lei, Jian Yu, and Shuigeng Zhou , Vol. 2, 2007, pp641 - 646

[11] Mirko Navara "Triangular norms and conorms" (2007), Scholarpedia, 2(3):2398. doi:10.4249/scholarpedia.2398

[12] Murali Raj.M and Thangathamizh.R, "Fixed point theorems in revised fuzzy metric space”, http://dx.doi.org/10.17654/FS026020103.

[13] J. Musielak and W. Orlicz, "On modular spaces," Studia Mathematica, vol. 18, pp. 49-65, 1959.

[14] Olga Grigorenko, Juan joseMinana, Alexander Sostak, Oscar Valero "On tconorm based Fuzzy (Pseudo) metrics”, Axioms 2020, 9, 78;doi:10.3390/axioms9030078.

[15] Yonghong shen, “ on fuzzy modular space” Volume 2013, Article ID 576237, 8 pages http://dx.doi.org/10.1155/2013/576237

[16] Zadeh.L.A, “Fuzzy sets”, Inform. Control, 8 (1965),338-353. 
